\title{
PREVALENCIA DE ALTA SOSPECHA DEL SÍNDROME DE APNEA OBSTRUCTIVA DEL SUEÑO EN PREESCOLARES DE BUCARAMANGA
}

\author{
${ }^{1}$ Marly Blanco C., ${ }^{1}$ Maria Teresa Santos R., ${ }^{1}$ Laura Cristina Madrigal R., ${ }^{2}$ Diana Marina Camargo L. \\ ${ }^{1}$ Estudiante X Semestre, Facultad de Odontología, U. Santo Tomás, ${ }^{2} M$ Sc Epidemiología, Profesora auxiliar \\ Escuela de Fisioterapia, U. Industrial de Santander
}

Autor responsable de correspondencia: Dra. Diana Marina Camargo L. Correo electrónico: diana_nico@yahoo.es

Premio al Mejor Trabajo de Investigación de Pregrado en el Área de Ortodoncia y Crecimiento y Desarrollo en el XIV Encuentro Nacional y III Latinoamericano de Investigación Odontológica, Septiembre de 2003.

\section{RESUMEN}

Objetivos: Determinar la prevalencia de alta sospecha del síndrome de apnea obstructiva del sueño en pre-escolares, y evaluar la consistencia interna del instrumento utilizado para su determinación.

Materiales y métodos: Se desarrolló un estudio observacional descriptivo en una población de 900 individuos con edades entre los 3 y 6 años, matriculados en instituciones públicas y privadas del municipio de Bucaramanga, entre febrero y mayo de 2003. Se seleccionaron al azar 60 concentraciones escolares; en cada una se recolectaron entre 15 y 30 encuestas dobles, es decir, una dirigida a los padres de familia y otra al profesor del grupo seleccionado. Para la evaluación de posibles asociaciones se aplicó la prueba de $\mathrm{X}^{2}$; la consistencia interna se determinó con el alpha de Chronbach, con un $\alpha=0.05$. Resultados: Se encontró una prevalencia de alta sospecha de apnea de 0.5\%, IC 95\% (0.14 - 1.3) y moderada sospecha de $3.55 \%$, IC 95\% (2.4 - 5.1). La adopción de diversas posturas durante el sueño y el ronquido fueron del 73.9\% y del 18\%, respectivamente. No se encontraron asociaciones significativas entre género y edad con alta sospecha de apnea.

Conclusiones: La apnea del sueño implica un manejo interdisciplinario; su detección precoz favorece una intervención adecuada y oportuna, lo cual evitará futuras complicaciones y contribuirá a mejorar la calidad de vida de los pre-escolares. [Blanco M, Santos MT, Madrigal LC, Camargo DM. Prevalencia de alta sospecha del síndrome de apnea obstructiva del sueño en preescolares de Bucaramanga. Ustasalud Odontología 2003; 2: 65 -72]

PALABRAS CLAVE: Apnea obstructiva, pre-escolares, resistencia vías aéreas superiores, prevalencia.

\section{Prevalence of the obstructive sleep apnea syndrome in pre-school children from Bucaramanga.}

\begin{abstract}
Purposes: To determine the prevalence of obstructive sleep apnea syndrome (OSAS) in a sample of pre-school children and to evaluate the internal consistence of the instrument used.

Materials and methods: A descriptive study was done to determine the prevalence of obstructive sleep apnea syndrome in 900 pre-school children. The age range was 3 to 6 years old. The children were registered in public and private institutions from Bucaramanga, from february til may 2003. Sixty (60) schools were selected randomly, in each institution were gathered 15 or 30 double surveys. One directed to the teacher of the selected group and the other one to the relatives. Chi square test and Chronbach alpha were applied to evaluate associations. 0.05 was determined as significant.

Results: The prevalence of high suspect of obstructive sleep apnea syndrome was confirmed in $0.5 \%$, IC 95\% (0.14 - 1.3$)$ and moderate in 3.55\%, IC 95\% (2.4 - 5.1). Nocturnal changes postures and snoring were $73.9 \%$ and $18 \%$, respectively. No associations were found between age and gender with OSAS (Obstructive sleep apnea syndrome).

Conclusions: The OSAS require an interdisciplinary managing; early detection helps a suitable and opportune treatment, that avoid future complications and improves the pre-scholars quality of life.
\end{abstract}

KEY WORDS: Obstructive sleep apnea syndrome, pre-scholars, upper airway resistance, prevalence. 


\section{INTRODUCCIÓN}

La prevalencia de desórdenes de sueño en menores de 5 años oscila entre 25 y 30\%; y, entre ellos, la apnea obstructiva del sueño afecta entre el 1 y $3 \%$ de esta población. ${ }^{1}$

La apnea se establece cuando no existe intercambio del flujo aéreo a nivel de las vías aéreas superiores, a pesar de la persistencia de estímulos a nivel central, como lo demuestra la movilidad diafragmática y la acentuación progresiva de la actividad de los músculos respiratorios. Esta entidad clínica es bien conocida en la población adulta; pero sólo hace poco ha tomado gran interés su estudio en la población infantil. ${ }^{1,2}$

Se estima, con base en algunos estudios epidemiológicos, que esta entidad tiene una prevalencia entre un 2 y $4 \%$ de la población adulta, predomina en sujetos masculinos de mediana edad y obesos en el mundo industrializado y de 1 - 2\% de la población general en Estados Unidos y Japón, aunque se sugiere que hay muchos casos no diagnosticados en estos dos países. En Cuba se encontró una frecuencia de un $4 \%$, en una muestra poblacional. ${ }^{1}$

En la población de menores de 6 años, la prevalencia del Síndrome de Apnea Obstructiva del Sueño (SAOS) es dificil de calcular, porque los estudios publicados usan diferentes métodos e interpretaciones en el análisis del polisomnograma (PSG) y otro tipo de pruebas, con cifras entre 0.7 y $10.3 \% .^{3,4}$

Al analizar la razón por género no hay acuerdo en los datos registrados en la literatura revisada. Las investigaciones realizadas en el Reino Unido han estimado una razón de 1:1 para hombres y mujeres; por otra parte, los estudios norteamericanos muestran una razón de 2:1, también para hombres y mujeres. ${ }^{4}$

Diversas publicaciones evidencian las complicaciones a que puede llevar este trastorno insidioso, de progresión gradual, que suele ser crónico, las cuales incluyen anormalidades en el crecimiento, desórdenes neurológicos, pulmonares y cardiovasculares, especialmente en casos severos, que ocasionan la muerte.,

En la literatura revisada no se han encontrado publicaciones relacionadas con la magnitud del problema en este grupo poblacional en Colombia ni instrumentos diseñados para su determinación. Por medio de esta investiga- ción se pretende determinar la prevalencia de la alta sospecha de $S A O S$ en la población preescolar de la ciudad de Bucaramanga y evaluar la consistencia interna del instrumento diseñado.

\section{MATERIALES Y MÉTODOS}

Se desarrolló un estudio observacional descriptivo; la población de estudio estuvo conformada por individuos entre tres y seis años, matriculados en instituciones públicas 0 privadas del municipio de Bucaramanga, entre febrero y mayo de 2003.

Se excluyeron instituciones ubicadas en sectores peligrosos de la ciudad e instituciones rurales debido a inconvenientes de accesibilidad geográfica.

Como criterios de inclusión se establecieron niños y niñas con edades entre los tres y seis años y la aceptación voluntaria de las instituciones, docentes y padres para responder la encuesta, en instituciones privadas y públicas del municipio de Bucaramanga.

Se aplicaron dos formularios, tipo encuesta, para la recolección de la información, uno dirigido a los padres y otro a los docentes. Las variables definidas consideraron las sociodemográficas generales, como: Género, edad, nivel de escolaridad, concentración escolar, jornada de estudio, estrato socioeconómico, períodos de estudio.

Adicionalmente, el instrumento incluyó cuatro atributos, de los cuales, los tres primeros fueron evaluados por los padres y el último por el profesor a cargo.

1. Antecedentes clínicos: Se evaluaron los signos y síntomas clínicos asociados con SAOS en la población infantil, como: enfermedades respiratorias (asma, rinitis, bronquitis), hipertrofia adenotonsilar, desviación del tabique, obstrucción de las fosas nasales y respiración oral.

2. Aspectos relacionados con el sueño: Se consideraron la adopción de diversas posturas durante el sueño, sudoración, ronquidos, pesadillas, enuresis, despertar intermitente, cefaleas matutinas, hipersomnolencia, cansancio, cambios de estado de ánimo.

3. Comportamiento diurno: Somnolencia diurna en varias situaciones, cefaleas matutinas, cansancio y cambios de estados de ánimo.

66

Ustasalud Odontología 2003; 2: 65 - 72

Blanco M. y col. 
4.Rendimiento académico: Cambios de estados de ánimo, falta de concentración, hiperactividad, disminución del rendimiento académico, atención limitada, pérdida de motivación.

\section{Análisis}

Inicialmente se describió la población de estudio en sus características sociodemográficas, se aplicaron medidas de tendencia central y dispersión o tablas de frecuencia, según la naturaleza, y distribución de cada variable. Las variables medidas en escala nominal se describieron en tablas de frecuencia. ${ }^{8}$

Adicionalmente, se estableció la frecuencia de las alteraciones del sueño y se calcularon sus intervalos de confianza del 95\%.

El análisis de la consistencia interna de los cuatro atributos y del instrumento, en general, se realizó aplicando un alpha de Chronbach. ${ }^{9}$

Para el análisis bivariado se estableció, como variable dependiente, alta sospecha de apnea y se eliminaron del análisis, los datos que correspondían a la opción de respuesta "No sabe", por lo cual quedaron finalmente 761 pre-escolares.

El procedimiento, similar al establecido por Netzer, ${ }^{10}$ en el cuestionario de Berlín para definir alto riesgo, se define a continuación: Inicialmente se totalizaron los puntajes de alteración en las cuatro categorías de variables definidas en el estudio, es decir, las alteraciones clínicas, aspectos relacionados con el sueño, comportamiento diurno y aspectos relacionados con el rendimiento académico. Los puntajes totales para cada una fueron:

a. Rendimiento académico: 0 - 9

b. Antecedentes clínicos: 0-6

c. Alteraciones del sueño: $\quad 0-6$

d. Comportamiento diurno: 0 - 6

Posteriormente se definieron cuatro categorías de alta sospecha, con base en el percentil 75 de la distribución para cada grupo de variables. Por lo anterior, se categorizaron así:

a. Rendimiento académico:

puntajes superiores a $2=$ alta sospecha

b. Antecedentes clínicos:

puntajes superiores a $1=$ alta sospecha c. Alteraciones del sueño:

puntajes superiores a $2=$ alta sospecha

d. Comportamiento diurno:

puntajes superiores a $2=$ alta sospecha

Finalmente, se sumaron las cuatro categorías, lo que determinó un rango entre cero y cuatro. Con base en estos datos se definió la escala: Baja sospecha para puntajes entre 0 y 1 ; moderada sospecha para puntajes iguales a 2 , y alta sospecha de apnea para los individuos con puntajes mayores o iguales a 3 .

El análisis de posibles asociaciones entre las variables sociodemográficas, se determinó con base en la alta sospecha, ya definida, aplicando una prueba de $\mathrm{X}^{2} .{ }^{11}$

Adicionalmente, se evaluaron posibles asociaciones entre la presencia de ronquido nocturno, con los otros antecedentes clínicos y el rendimiento académico; se aplicó la prueba ya mencionada.

La base de datos se elaboró en Excel ${ }^{12}$ y el análisis en STATA $6.0^{13}$ considerando un nivel de significancia $\alpha=$ 0,05 .

Esta investigación se realizó considerando la Resolución 008430, de 1993, del Ministerio de Salud de Colombia, que establece en el Título II de la investigación en seres humanos en su artículo 11, que esta investigación se considera sin riesgo, puesto que el estudio emplea técnicas y métodos de investigación documental en los que no se realiza ninguna intervención o modificación intencionada de las variables biológicas, fisiológicas, psicológicas 0 sociales de los individuos que participan en el estudio. Se solicitó el consentimiento informado tanto de la institución como de los padres de familia.

\section{RESULTADOS}

\section{Descripción general.}

En total se evaluaron 900 preescolares, 468 (52\%) del género masculino, con un rango de edad de 2 a 6 años y una mediana de 4 años.

La distribución por tipo de concentración escolar mostró un $60 \%$ de instituciones privadas, con predominio de la jornada de la mañana, correspondiente a 576 escolares (64\%), y, en segundo lugar, en la tarde 295 (32.8\%); el excedente tenía doble jornada. 
En cuanto a los años de estudio del escolar, el grupo de mayor predominio fue de 1 año, con 431 (48\%); luego, 2 años, con 326 (36\%); y, en tercer lugar, 3 años, con 100 (11.1\%).

El estrato socioeconómico más frecuente fue el 4, con 310 (34.4\%); luego el estrato 3, con 194 (22\%), y el estrato 2 con $185(21 \%)$.

Las observaciones registradas mostraron 4 niños con adenoides grandes; 5 niños sufren rinitis; 1 sufre de afonía repentina y expectora; 1 con bruxismo; 2 con amigdalitis; 1 con síndrome nefrítico; 3 son respiradores orales; 1 con sudoración nocturna; 2 con síndrome de Down; 3 que toman medicamentos para la epilepsia; 1 con hidrocefalia; 1 es autista y tiene problemas de motricidad, y 1 con problemas de aprendizaje y lenguaje.

Para establecer las cifras de prevalencia, se eliminaron de la base de datos los registros cuyas respuestas se codificaron en la categoría de "no sabe", para las preguntas relacionadas con antecedentes clínicos y levantadas nocturnas, de aspectos relacionados con el sueño. Por lo tanto, la base para la prevalencia fue de 761 individuos.

En la Tabla 1, se presentan los resultados de los antecedentes clínicos conocidos por los padres de familia. De esto resulta que 56 (7.3\%) sufren de asma; 76 (9.9\%), de rinitis; 44 (5.8\%), de bronquitis; 118 (15.5\%), de dolor de garganta; 85 (11.2\%) viven con la nariz tapada; 9 (1.2\%) tienen la nariz desviada; y 102 (13.4\%) respiran por la boca.

En la Tabla 2, se presentan los resultados de los aspectos relacionados con el sueño, percibidos por los padres de familia: 87 (11.4\%) niños se despiertan en forma constante durante la noche; 117 (15.4\%) acuden a sus padres por tener pesadillas; 137 (18\%) roncan; 562 (73.9\%) adoptan diversas posturas durante el sueño; 312 (41\%) tienen sudoración nocturna; 79 (10.4\%) sufren de enuresis nocturna.

Los resultados del comportamiento del niño, percibido por los padres de familia durante el día, se presentan en la Tabla 3: 70 (9.2\%) se quedan dormidos leyendo; $264(34,7 \%)$, viendo televisión; 77 (10.1\%), en un lugar público; 23 (3\%), sentados hablando con alguien; 112 (14.7\%), sentados después de haber comido; 49 (6.4\%, sufren de dolor de cabeza durante el día; 22 (8.1\%), sienten cansancio cuando realizan alguna actividad; 186 (24.4\%), cambios de estado de ánimo.
La Tabla 4 presenta los resultados de los aspectos relacionados con el comportamiento y el rendimiento académico del niño percibido por los profesores: 18 (2.4\%) nunca permanecen concentrados durante la clase; $69(9.1 \%)$ no están motivados para realizar las actividades; 82 (10.8\%) no comprenden ni desarrollan con facilidad las actividades; 77 (10.1\%) presentan disminución en la asimilación de los contenidos vistos en clase; 77 (10.1\%) presentan disminución en su rendimiento académico, y 62 (8.2\%) presentan cambios en su estado de ánimo.

\section{Análisis de consistencia interna}

Al aplicar el alpha de Chronbach se encontraron consistencias internas de $0.76,0.57,0.45$ y 0.41 para rendimiento académico, antecedentes clínicos, alteraciones del sueño y comportamiento diurno, respectivamente.

Se tomó como base el rendimiento académico, al adicionar los antecedentes clínicos $\alpha=0.67$, más las alteraciones del sueño $\alpha=0.57$ y con el comportamiento diurno $\alpha=0.62$, equivalente a la consistencia interna del instrumento completo.

\section{Categorías de apnea}

Con base en la clasificación establecida y siguiendo la metodología de Netzer ${ }^{10}$ en el formulario de Berlín, se establecieron cuatro categorías de sospecha de apnea (Tabla 5), con sus intervalos de confianza del 95\%; encontrando en la categoría de alta sospecha, una prevalencia de $0.5 \%$, IC $95 \%$ (0.14 - 1.3) y moderada sospecha de $3.55 \%$, IC 95\% (2.4 - 5.1). Al analizar la razón hombre 0 mujer en cada categoría, se encontró una razón de 3:1 y de 2:1, respectivamente.

\section{Análisis bivariado}

\section{Alta sospecha de apnea:}

Los resultados del análisis divariado, de alta sospecha de apnea por género y edad, se muestran en la Tabla 6 . No se encontraron asociaciones estadísticamente significativas.

\section{Ronquido nocturno:}

Los hallazgos del análisis bivariado para ronquido nocturno, según las variables sociodemográficas se presentan en la Tabla 7. A excepción de la edad, el ronquido se asoció en forma significativa con los antecedentes clínicos y con el género masculino. La razón hombre : mujer fue de 1.4: 1. 
Tabla 1. Descripción de los antecedentes clínicos registrados por padres (n: 761).

\begin{tabular}{lccc}
\hline \multicolumn{1}{c}{ VARIABLE } & No & $\%$ & IC 95\% \\
\hline Asma & 56 & 7,3 & $5.5-9.2$ \\
Rinitis & 76 & 9,9 & $7.8-12.2$ \\
Bronquitis & 44 & 5,8 & $4.1-7.4$ \\
Dolor de garganta & 118 & 15.5 & $12.9-18.1$ \\
Nariz tapada & 85 & 11.2 & $8.9-13.4$ \\
Desviación de la nariz & 9 & 1.2 & $0.4-1.9$ \\
Respirador oral & 102 & 13,4 & $10.9-15.8$ \\
\hline
\end{tabular}

Tabla 2. Descripción de los aspectos relacionados con el sueño registrado por los padres (n: 761).

\begin{tabular}{lccc}
\hline \multicolumn{1}{c}{ VARIABLE } & No & $\%$ & IC 95\% \\
\hline Despertar nocturno & 87 & 11.4 & $9.2-13.7$ \\
Pesadillas & 117 & 15.4 & $12.8-17.9$ \\
Ronquidos & 137 & 18 & $15.2-20.7$ \\
Adopción de posturas & 562 & 73.9 & $70.7-77.0$ \\
Sudoración & 312 & 41 & $37.5-44.5$ \\
Enuresis nocturna & 79 & 10.4 & $8.2-12.6$ \\
\hline
\end{tabular}

Tabla 3. Descripción del comportamiento del niño durante el día registrado por los padres (n: 761).

\begin{tabular}{lccc}
\hline \multicolumn{1}{c}{ VARIABLE } & No & $\%$ & IC 95\% \\
\hline Somnolencia al leer & 70 & 9.2 & $7.1-11.3$ \\
Somnolencia viendo televisión & 264 & 34,7 & $31.3-38.1$ \\
Somnolencia en lugar público & 77 & 10,1 & $7.9-12.2$ \\
Somnolencia hablando & 23 & 3.0 & $1.8-4.2$ \\
Somnolencia después de comer & 112 & 14.7 & $12.2-17.2$ \\
Cefaleas matutinas & 49 & 6.4 & $4.6-8.1$ \\
Cansancio & 22 & 8.1 & $6.2-10.1$ \\
Cambios de estado de ánimo & 186 & 24.4 & $21.4-27.5$ \\
\hline \hline
\end{tabular}

Tabla 4. Descripción de los aspectos relacionados con el comportamiento y rendimiento académico del niño registrado por los profesores (n: 761).

\begin{tabular}{lccc}
\hline \multicolumn{1}{c}{ VARIABLE } & No & $\%$ & IC 95\% \\
\hline Nunca se concentra en clase & 18 & 2.4 & $1.4-3.7$ \\
Ausencia de motivación en clase & 69 & 9.1 & $7.0-1.1$ \\
Palta de comprensión para el desarrollo de actividades & 82 & 10.8 & $8.5-13.0$ \\
Disminución en la asimilación de contenidos & 77 & 10.1 & $8.0-12.3$ \\
Disminución rendimiento académico & 77 & 10.1 & $8.0-12.3$ \\
Cambios de estado de ánimo (irritado, inquieto, de mal & 62 & 8.2 & $6.3-10.3$ \\
humor en el dia) & & & \\
\hline
\end{tabular}

Tabla 5. Prevalencia de sospecha de apnea.

\begin{tabular}{lccc}
\hline \multicolumn{1}{c}{ Categoria } & No & \% & IC 95\% \\
\hline Alta & 4 & 0.53 & $0.14-1.3$ \\
Moderada & 27 & 3.55 & $2.4-5.1$ \\
Baja & 94 & 12.35 & $10.1-14.9$ \\
\hline Sin & 636 & 83.6 & $80.7-86.1$ \\
\hline
\end{tabular}

Tabla 6. Evaluación de posibles asociaciones entre género y edad con alta sospecha de apnea.

\begin{tabular}{rccc}
\hline & \multicolumn{3}{c}{ Sospecha de apnea } \\
\cline { 2 - 3 } Variable & Alta & Moderada + Baja + Sin & p \\
& $(n: 4)$ & $(n: 757)$ & 0.35 \\
Género masculino & 3 & 389 & 0.35 \\
Promedio de edad & 4.2 & 4.8 & \\
\hline
\end{tabular}


Tabla 7. Evaluación de posibles asociaciones entre género, edad, antecedentes clínicos y alteraciones de comportamiento con ronquido nocturno.

\begin{tabular}{|c|c|c|c|}
\hline \multirow{3}{*}{ Variable } & \multicolumn{2}{|c|}{ Ronquido } & \multirow{3}{*}{ p } \\
\hline & Si & No & \\
\hline & $n: 137$ & $n: 624$ & \\
\hline Género masculino & 81 & 311 & 0.05 \\
\hline Promedio de edad & 42 & 4.3 & 0.50 \\
\hline Asma & 19 & 37 & 0.01 \\
\hline Rinitis & 24 & 52 & 0.01 \\
\hline Bronquitis & 14 & 30 & 0.01 \\
\hline Dolor de garganta frecuente & 31 & 87 & 0.01 \\
\hline Nariz tapada sin gripe & 37 & 48 & $<0.0001$ \\
\hline Nariz desviada & 6 & 3 & $<0.0001$ \\
\hline Respira con la boca abierta & 51 & 51 & $<0.0001$ \\
\hline Cefalea diurna & 18 & 31 & $<0.0001$ \\
\hline Cambios de estados de ánimo & 47 & 139 & 0.03 \\
\hline
\end{tabular}

\section{DISCUSIÓN}

Los desórdenes del sueño son comunes en la infancia y pueden afectar múltiples aspectos de la vida del niño y de otros miembros de la familia. De ahí la importancia de evaluarlos con una historia clínica completa, que permita la interrelación de diversos factores. ${ }^{1}$

Los estudios epidemiológicos de apnea del sueño son dificiles de realizar, puesto que el diagnóstico confirmatorio requiere una polisomnografía, prueba dispendiosa y de alto costo, que hacen dificil su aplicación en estudios con base poblacional. ${ }^{14}$ Por lo anterior, se recurre a la aplicación de encuestas, en las cuales se determina la frecuencia de los signos y síntomas más frecuentemente asociados con el Síndrome de Apnea Obstructiva del Sueño y otro tipo de pruebas diagnósticas, con variables niveles de validez. Adicionalmente, aún no hay acuerdo en la exactitud del polisomnograma para el diagnóstico del SAOS. ${ }^{3,15-17}$

La importancia del diagnóstico precoz de la apnea del sueño en la población infantil radica en la posibilidad de plantear la mejor estrategia de intervención, en forma oportuna, ya que la apnea del sueño no tratada puede resultar en complicaciones serias, como discapacidad neurocognitiva, problemas de comportamiento, bajo rendimiento escolar, disminución de la calidad de vida, cor pulmonar y muerte; en la vida adulta media se ha encontrado asociada con hipertensión arterial, entre otros problemas cardiovasculares. ${ }^{3,}$ 5-7, 18-20
Los estudios previos estiman que la prevalencia del $S A O S$ se da entre el 1 y $3 \%$, en los niños de edad pre-escolar y escolar., 16, 19, 21,22 Los hallazgos de este estudio muestran una prevalencia de alta sospecha de apnea de $0.5 \%$, IC $95 \%$ (0.1 - 1.3) y moderada sospecha de apnea de $3.55 \%$, IC 95\% (2.4 - 5.1). Datos similares, si se consideran las dos categorías en conjunto. Sin embargo, es importante mencionar que la mayoría de los estudios no cuentan con una base poblacional, lo que probablemente alteraría los resultados, si se aplicara la prueba de la polisomnografía en esta población.

Los hallazgos de estudios anteriores muestran una distribución equitativa por género. ${ }^{7,16,18}$ Nuestros datos muestran una razón hombre:mujer de 3:1 en la alta sospecha de apnea y de 2:1 en moderada sospecha del Síndrome de Apnea Obstructiva del Sueño. Una posible explicación de este fenómeno puede hallarse en la subjetividad inherente a las preguntas consideradas en la encuesta, diligenciada por los padres y profesores, aspecto que sólo podría demostrarse con la polisomnografía, como ya se ha demostrado en otras investigaciones. ${ }^{14,23 .}$

La relevancia de disponer de un instrumento para tamizaje ha sido establecida previamente. ${ }^{23}$ Dicho estudio, sin embargo, concluye que la historia clínica sola no puede discriminar entre el ronquido primario y el Síndrome de Apnea Obstructiva del Sueño.

Por lo anterior, y con el fin de ampliar los signos y síntomas del Síndrome de Apnea Obstructiva del Sueño, se establecieron, para este estudio, cuatro atributos, que 
probablemente están asociados con alta sospecha de apnea. Los hallazgos del análisis de consistencia interna muestran cómo las variables relacionadas con rendimiento académico, presentan el mayor alpha de Chronbach (0.76); los ítems incluidos han sido determinados previamente como signos del SOAS en la población infantil. 1,3, 4, 18, 20, 22

Los antecedentes clínicos que incluyen dolor de garganta, respiración oral, nariz tapada, rinitis y asma, son todos signos asociados con la apnea del sueño en población infantil; lo que sugiere que la alergia es común en niños pequeños con ronquido primario y $S A O S$. Una posible explicación es que los signos alérgicos se comparten en las vías aéreas superiores con la apnea, lo cual debe alertar a los clínicos para descartar cualquiera de los dos diagnósticos. ${ }^{24}$ La consistencia interna de este atributo fue de 0.57, moderada, y ocupó el segundo lugar.

Las variables relacionadas con el atributo del sueño y comportamiento diurno mostraron la más baja consistencia interna con alphas de 0.45 y 0.41 . Sin embargo, es importante destacar que todos los ítems incluidos ya se han encontrado asociados con la apnea del sueño.

La validez del constructo medida con el alpha de Chronbach, en el instrumento completo, fue de 0.62 , que se categoriza como moderada.

Estos hallazgos plantean un inicio en la propuesta y evaluación de instrumentos de tamizaje en la población infantil, para preseleccionar individuos con alta sospecha de apnea de sueño, cuyo diagnóstico definitivo probablemente sería establecido con una polisomnografía, prueba de oro reconocida hasta la fecha, contra la cual se evaluarían las propiedades psicometricas de cualquier instrumento de medición. ${ }^{25,26}$ Este análisis es una de las fortalezas de este trabajo, pues en ninguno de los artículos revisados se ha aplicado con anterioridad.

La evaluación de las asociaciones entre género y edad, con alta sospecha de apnea, no fue estadísticamente significativa; probablemente esto se deba a la baja frecuencia de individuos en esta categoría. Sin embargo, al totalizar moderada y alta, tampoco se detectaron cambios en la significancia estadística.

El ronquido es uno de los principales signos asociados al Síndrome de Apnea Obstructiva del Sueño, cuya prevalencia fue de 18\% (137/761), IC 95\% (15.2 - 20.7), superior a la cifra registrada en otros estudios, que muestran una prevalencia entre 7 y $12 \% .{ }^{27-31}$ Posiblemente esto se deba a la subjetividad ya planteada. Sin embargo, un estudio de corte transversal, realizado en Hong Kong muestra cifras similares a las de este trabajo 14.5\%, IC 95\% (10 20), con una razón hombre: mujer de 1.4: 1.27

La asociación entre ronquido y alteraciones de comportamiento, al igual que las enfermedades alérgicas en la población pediátrica, detectada en este estudio, (Tabla 9) ha sido demostrada en varias publicaciones. ${ }^{5,24,27}$ Es importante su detección, ya que incrementa el riesgo del Síndrome de Apnea Obstructiva del Sueño por efecto de la resistencia de la vía aérea superior y predispone a una obstrucción parcial o completa durante el sueño. ${ }^{24}$ Igualmente, su detección precoz favorece una intervención adecuada y oportuna, con lo que contribuye, en una forma significativa, a mejorar la calidad de vida del infante.

Hay que tener en cuenta que los cuestionarios clínicos son de dificil aceptación por su baja especificidad y sensibilidad; pero hacen parte del diagnóstico para seleccionar la población, para que sea remitida y valorada y así diagnosticar el Síndrome de Apnea Obstructiva del Sueño, mediante la polisomnografía.

El Síndrome de Apnea Obstructiva del Sueño es una enfermedad que implica un manejo interdisciplinario. Un diagnóstico correcto ayudará a evitar futuras complicaciones y sobre todo a devolver la salud a los niños que presentan esta enfermedad. El odontólogo, el odontopediatra y el ortodoncista, pueden ser los primeros profesionales del equipo de salud en identificar $a$ un individuo con riesgo de tener apnea del sueño, pues hacen parte de los profesionales del área estomatológica con mayor entrenamiento para la valoración de la vía aérea superior. Por lo tanto, deben recibir entrenamiento en los signos y síntomas asociados con el síndrome, de modo que puedan identificar al paciente, remitirlo y/o tratarlo, si es pertinente. ${ }^{32-33}$

\section{BIBLIOGRAFÍA}

1. Ward T, Thornton BA, Mason II. Sleep disorders in children. Nurs Clin N Am 2002; 37: 693 - 706.

2. García X, Damiani S, De La Osa J. Síndrome de apnea obstructiva del sueño. Conocimientos importantes para todo profesional de la salud. Rev Cubana Med Gen Integr 1999; 15: 562 -569. 
3. Schechter MS, Technical report: diagnosis and management of childhood obstructive sleep apnea syndrome. Pediatrics 2002; 109: 1 20.

4. Steffan M. Sleep apnea. Medicine Journal 2002; 3: 1 - 15.

5. Katzenmeyer WK. Adult and pediatric obstructive sleep apnea. Grand Rounds presentation, UTMB, Dept. of otolaryngology, Material prepared by resident physicians in partial fulfillment of educational requirements for postgraduate training program of the UTMB Department. June 5, 2002.

6. Vázquez C, Meza S, Méndez J. Respiración y sueño. Caso clínico. Rev Inst Nal Enf Resp Mex 2000; 13: 163 - 169.

7. Barret M. Obstructive sleep apnea syndrome in children. American Family Physician 2002; 66: 1338 - 1340.

8. Pagano M, Gauvreau K. Principles of biostatistics. Duxbury Press, Belmont Ca, 1993.

9. Nunnally JC, Bernstein IJ. Teoría Psicométrica. 3ra. Ed. México: McGraw Hill; 1995.

10. Netzer N, Stoohs R. Using the Berlin questionnaire to identify patients at risk for the sleep apnea syndrome. Annals of Internal Medicine. 1999; 131: 485491.

11. Siegel S, Castellan NJ. Nonparametric statistics for the behavioral sciences. 2nd. Ed. Singapore: McGraw Hill; 1988.

12. Microsoft Excel, 5.0, Microsoft Corporation, 1997.

13. StataCorp. 1999. Stata Statistical Software: Release 6.0. College Station, TX: Stata Corporation.

14. Puvandendran K, Goh KL. From snoring to sleep apnea in a Singapore population. Sleep Research Online 1999; 2: 11 - 14

15. Ross SD, Allen IE, Harrison KJ. Review: Screening test as not are accurate as overnight polysomnography for the diagnosis of adult sleep apnea. Evidence Based Medicine 2000; 5: 61.

16. Pelletier-Fleury N, Lanoé JL. Equivalence versus pragmatic trials for the economic evaluation of information and communication technologies; the case of polysomnography under telesurveillance in the diagnosis of sleep apnea syndrome. Health policy 2001; 57: 225 234.

17. Teculescu DB, Montaunt-Verient B, Hannhart B, Virion JM, Cornette A, Michaely JP. Breathing pauses during sleep. ¿Can a non-invasive ENT examination help identify subjects at risk in epidemiological settings? Medical Hypothesis 2001; 56: 653 - 656.

18. Lipton AJ, Gozal D. Obstructive sleep apnea syndrome. Medicine Journal 2002; 3: 1517.

19. Brunetti L, Rana S. Lospalluti ML, Pietrafesa A, Francavilla R, Fanelli M. Armenio L. Prevalence of obstructive sleep apnea syndrome in a cohort of 1207 children of southern Italy. Chest 2001; 120:1930 - 1935.
20. Chervin RD, Dillon JE, Archbold KH, Ruzicka DL. Conduct problems and symptoms of sleep disorders in children. J Am Acad Chil Adolesc Psychiatry 2003; 42: 201 - 208.

21. Sjostrom C, Lindberg E, Elmasry A, Hagg A. Kavarsudd K, Janson C. Prevalence of sleep apnea and snoring in hypertensive men: a population based study. Thorax 2002; 57: 602 - 607.

22. McNamara F, Sullivan CE. The genesis of adult sleep apnea in childhood. Thorax 2000; 55: 964 - 969.

23. Smeyers-Durán P. Téllez de Meneses M. Narcolepsia y síndrome de apnea obstructiva del sueño en el niño. Rev Neurol Clin 2000; 1 : 143 149.

24. Carroll JL, McColley SA, Marcus CL, Curtis S, Loughlin GM. Inability of clinical history distinguishes primary snoring from obstructive sleep apnea syndrome in children. Chest 1995; 108: 610 - 618.

25. McColley SA, Carroll JL. Curtis S, Loughlin GM, Sampson HA. High prevalence of allergic sensitization in children with habitual snoring and obstructive sleep apnea. Chest 1997; 111: 170 - 173.

26. Coste J, Fermanian J, Venot A. Methodological and statistical problems in the construction of composite measurement Scales: A survey of six medical and epidemiological. Journal Statistics in Medicine 1995; 14: 331 - 345.

27. Kirshner B, Guyatt G. Methodological framework for assessing health indices. J Chronic Dis 1985; 38: 27 - 36.

28. Ng D K-K, Kwok KL, Poon G, Chau K-W. Habitual snoring and sleep bruxism in a paediatric outpatient population in Hong Kong. Singapore Med J 2002; 33: 554556.

29. Gislason T, Benediktsdóttir B. Snoring, apneic episodes and nocturnal hypoxemia among children 6 month to 6 years old. An epidemiologic study of lower limit of prevalence. Chest 1995; 107: 963 - 966.

30. Hui DSC, Chan JKW, Ho ASS, Choy DKL, Lai CKW, Leung RC. Prevalence of snoring and sleep -disordered breathing in a student population. Chest 1999; 116: 1530 -1536.

31. Castronovo V. Zucconi M, Nosetti L, Marazzini C, Hensley M, Veglia F, Nespoli L, Ferini-Strambi L. Prevalence of habitual snoring and sleep - disordered breathing in preschool-aged children in an Italian community. J Pediatr 2003; 142: 377 - 382.

32. Vila Morales D, Garmendia Hernández G, Morales García N, Correa Mozo B. Síndrome de apnea obstructiva del sueño. Fisiopatología y Diagnóstico. Rev Cubana Ortod 2001; 16: 69 - 75.

33. Friedlander AH, Friedlander IK, Pogrel MA. Dentistry's role in the diagnosis and co-management of patients with sleep apnoea/hyponnoea syndrome. Br Dent J 2000; 189: 76 - 80.

34. Friendlander AH, Walker LA, Friendlander IK, Felsenfeld AL. Diagnosing and co managing patients with obstructive sleep apnea syndrome. JADA 2000; 131: 1178-1182. 